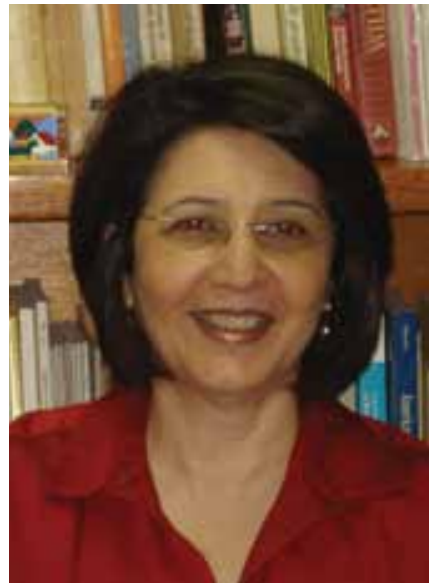

\title{
Educación General y Aprendizaje Liberal. Principios de prácticas efectivas
}

\author{
Ana Margarita Haché *
}

Resumen: Se reseña el libro General Education and Liberal Learning: Principles of Effective Practice. Este libro explora elementos comunes a los programas de Educación General y analiza cómo esos programas promueven el aprendizaje liberal, aspecto esencial para la educación del siglo veintiuno. La publicación expone los cambios que han ocurrido en los programas de Educación General, con énfasis en la Educación Superior. Se discute, también, cómo las instituciones pueden mejorar sus prácticas en estos programas a través de reportar ejemplos exitosos. El libro es útil para los comités de currículum y para todos los grupos que trabajen en evaluar programas de Educación General.

\begin{abstract}
This is a book review of General Education an Liberal Learning: Principles of Effective Practice. This book explores elements common to strong general education programs and examines how strong programs support liberal learning outcomes essential to success in the twenty-first century. The publication surveys the changes that have occurred in general education programs - and more broadly in higher education. The publication discusses also how institutions may improve their general education practices and provides numerous examples of successful practices. The book is useful for curriculum committees and any group of professionals that work on the assessment of General Education programs.
\end{abstract}

Este libro es auspiciado por la Asociación Americana de Colegios ${ }^{1}$ y Universidades (AAC\&U). Tiene como autor principal a Paul L. Gaston junto con una serie de colaboradores Elizabeth Clark, Ann S. Ferren, Peggy Maki, Terrel I. Rhodes, Karen Maitland Schelling y Dwight Smith. Consta de cuatro partes, con la siguiente estructura:

- Primera parte: Educación General, educación liberal

- Introducción

- Capítulo uno: Imperativos y motores del cambio

- Segunda parte: La Educación General como piedra angular

- Capítulo 2: Principios de los sólidos programas de Educación General
- Tercera parte: Prácticas efectivas en la Educación General

- Capítulo 3: Intencionalidad

- Capítulo 4: Alineación con las especialidades

- Capítulo 5: Pedagogía efectiva

- Capítulo 6: Evaluación

- Cuarta parte: La sostenibilidad de los programas de Educación General

-Capítulo 7: Compromiso institucional

En él se presenta, con la ayuda de diferentes autores, especialistas en el tema, los principios que caracterizan a los sólidos programas de Educación General en los Estados Unidos de América. Sus argumentos se basan en conceptos que definen a la Educación General en la actualidad y la hacen fun-
Palabras clave

Educación General, Aprendizaje

Liberal, Educación Superior,

diseño curricular

\section{Key Words}

General Education, Liberal

Learning, Higuer Education, curriculum design
${ }^{1}$ La autora ha traducido la palabra inglesa Colleges como Colegios en español.

"Magíster en Lingüística Hispánica, University of Florida. Directora del Centro de Desarrollo Profesoral de la Pontificia Universidad Católica Madre y Maestra, Campus de Santiago en el período 2000-2010. Forma parte del Consejo Asesor del Cuaderno de Pedagogía Universitaria publicado por esta Universidad. Ha sido autora de numerosos artículos y co-autora de libros en el área de lingüística aplicada a nivel nacional e internacional. Es asesora del Ministerio de Educación Superior, Ciencia y Tecnología de la República Dominicana en la evaluación quinquenal de instituciones de Educación Superior. Para contactar a a la autora: ahache@pucmmsti.edu.do

Encuentre el texto en "Los Estudios Generales en el currículum universitario" http://www.pucmm.edu.do/STI/campus/CDP/ComunicaciónPublicaciones/Paginas/CuadernodePedagogiaUniversitaria.aspx

Cuaderno de Pedagogía Universitaria Año 7/ N. 14 / julio - diciembre 2010 / Santiago, Rep. Dom.: PUCMM / pp.38-43 
damental para la consecución del aprendizaje liberal. Estos argumentos están avalados con ejemplos extraídos de la realidad de la educación superior en ese país. A pesar de que su particular contexto, las ideas expuestas pueden resultar iluminadoras para toda institución que tenga como misión la formación de seres humanos integrales que vivencien una ciudadanía plena desde el plano personal y profesional.

Para los fines de esta reseña, se ha ido resumiendo cada capítulo del libro priorizando las ideas que representan el sentido global del texto; además, se han ponderado y valorando los argumentos para dar al lector una visión de las fortalezas y debilidades de este material bibliográfico.

La introducción de este libro posiciona el tema de la Educación General a partir de los principios que fueron identificados como promotores de una Educación General efectiva y que aparecieron en el estudio de la Asociación Americana de Colegios y Universidades (AAC\&U) de 1994 titulado "Bases sólidas: doce principios para efectivos programas de Educación General".

Esta sección es de la autoría de Terrel L. Rhodes, quien lo sitúa en una época en que la Educación General está recibiendo renovada atención debido a diferentes factores. Primero, en algunas instituciones, estos cursos están siendo cuestionados a medida que las universidades luchan con la crisis financiera. Segundo, investigaciones realizadas por la AAC\&U indican que los empleadores buscan egresados con competencias generales tales como habilidades comunicativas, pensamiento crítico, aplicación de conocimientos a contextos reales, resolución de problemas, trabajo en equipo, innovación y creatividad, toma de decisiones éticas y conceptos y desarrollos en ciencia y tecnología. Tercero, las instituciones de educación superior norteamericanas están observando con atención la reforma europea de Bolonia que fija en tres años el grado universitario. Cuarto, numerosos educadores a nivel internacional reconocen que el egresado norteamericano parece ser más innovador y capaz de transferir el conocimiento a través de las disciplinas y las fronteras conceptuales que el de otros sistemas educativos.

Para el autor, algunos de los hechos antes mencionados se convierten en evidencias a favor de la eficiencia de un sistema educativo que asigna una función importante a la Educación General. Sin embargo, aboga por la necesidad de repensar los enfoques para la enseñanza y el aprendizaje en la Educación General. De esta forma, cobra importancia este nuevo libro "Educación General y Educación Liberal" que retoma los principios expuestos en 1994 para incorporar investigaciones y experiencias llevadas a cabo en los últimos 15 años y así reafirmar el compromiso con una Educación General efectiva en el siglo XXI.

El capítulo 1 del libro que se reseña se titula: Imperativos y motores del cambio y lo escribe por Paul L. Gaston. Inicia con una contundente cita que sirve de hilo conductor a través de todo el libro: para garantizar que el estudiantado reciba todos los resultados de una educación liberal, se requiere del apoyo que solo un efectivo programa de Educación General puede ofrecer. En este sentido, examinar cuidadosamente las formas exitosas que algunas instituciones han logrado con este tipo de educación, contribuiría a destacar los principios que la fortalecen y ayudarían a que de los Estudios Generales se eficienticen en todas las instituciones de educación superior donde existen.

En este capítulo se presentan los antecedentes que nutren esta publicación. Se trata de tres trabajos anteriores, con los cuales concuerda y toma como referencia. Uno es el publicado en 1994 titulado "Bases sólidas: doce principios para la eficacia de los programas de educación general". El segundo lo constituye "Mejores expectativas" que articula los propósitos de la educación liberal del siglo XXI y el tercero se refiere al informe estadounidense sobre el aprendizaje universitario para el nuevo siglo global. La conceptualización de la Educación General que se desprende de este libro incluye cuatro dominios del conocimiento:

- Conocimiento de las culturas humanas y del mundo físico y natural

- Habilidades intelectuales y prácticas

- Responsabilidad personal y social

- Aprendizaje integral y aplicado

Según Gaston, estos dominios podrían preparar al estudiantado para los retos del siglo XXI que se caracterizan por las siguientes tendencias: 1. Exigencias mundiales más demandantes

2. Un estudiantado diferente

3. La diversidad como valor educativo fundamental

4. Presiones económicas

5. La influencia de la tecnología

6. El imperativo de la evaluación

7. Los cambiantes roles y presiones del profesorado

Cabe destacar que este libro está pensado para un público que conoce lo que es la Educación General, pues salvo la explicitación de los cuatro dominios que fueron enunciados anteriormente, no aparece en sus páginas ninguna definición explícita sobre este tipo de enseñanza. Lo mismo ocurre con el aprendizaje liberal; en este caso, un lector poco avisado puede llegar a pensar que la única forma de alcanzar el aprendizaje liberal es a través de la Educación General. Sin embargo, hacia el final del libro Paul Gaston puntualiza que la Educación General es uno de los medios para acceder a esta forma de aprendizaje.

Aunque a través de la lectura de este interesante documento, el lector va formándose una definición de en qué consiste la Educación General, no sucede lo mismo con el concepto de aprendizaje liberal. Para comprender cabalmente su sentido, hay que indagar en otras fuentes su conceptualización. Por este motivo, es conveniente explicitar, al inicio de esta reseña, la definición que la propia Asociación Americana de Colegios y Universidades (AAC\&U) le atribuye a este término. Un aprendizaje verdaderamente liberal, según esta Asociación, prepara para vivir una vida responsable, productiva y creativa en un mundo dramáticamente cambiante. Fomenta una disposición hacia el aprendizaje permanente y una aceptación de responsabilidad por las consecuencias éticas de nuestras ideas y acciones. El aprendizaje liberal permite que entendamos los fundamentos del conocimiento y la investigación acerca de la naturaleza, la cultura y la sociedad; asimismo implica que los sujetos que incorporen este tipo de aprendizaje dominen las habi- 
lidades básicas de percepción, análisis y expresión, cultiven el respeto a la verdad, reconozcan de la importancia del contexto histórico y cultural y exploren las conexiones entre el aprendizaje formal, la ciudadanía, y el servicio a nuestras comunidades.

Teniendo estas concepciones incorporadas a nuestros esquemas de pensamiento, la construcción del sentido global del texto se facilita ya que es posible inferir, pues, que una Educación General sólida puede contribuir efectivamente a la adquisición del aprendizaje liberal.

Los principios de los sólidos programas de educación general conforman el segundo capítulo del libro reseñando y su autor es también Paul L. Gaston. En este acápite, Gaston nos advierte que los programas de Educación General considerados sólidos y eficientes no surgen por casualidad. Estos poseen una serie de cualidades que los distinguen. A saber:

1. encarnan y expresan una visión clara de la Educación General basada en un compromiso institucional sobre los beneficios de una educación liberal para todos los alumnos.

2. se benefician de un entendimiento coherente que permea a todos los grupos institucionales sobre la importante función que desempeña la Educación General.

3. presentan evidencias en todos los niveles curriculares de su preocupación por una pedagogía eficaz que exprese una búsqueda intencionada de objetivos de aprendizaje explícitos para formar ciudadanos responsables.

4. demuestran su aprecio por la función del programa co-curricular en la mejora y el mantenimiento de los logros obtenidos en el aula.

5. requieren y expresan un fuerte liderazgo desde muchos niveles de la comunidad educativa.

El autor se vale de una serie de instituciones para que el lector se forme un juicio pertinente sobre el significado de estas características; sin embargo, en ningún momento explica la metodología usada para la selección de esas instituciones. Aún más, será responsabilidad del lector comprobar si la puesta en práctica de estas características se cumple verdaderamente en las instituciones mencionadas. No obstante este vacío metodológico, el recurso de ejemplificar usando universidades y colegios de la realidad estadounidense confiere credibilidad a las ideas del autor y puede servir también de incentivos para otras instituciones de educación superior que busquen consolidar sus estudios generales.

A través de ejemplos de instituciones como North Carolina State University, West Point Military Academy y Brown University el lector llega a comprender qué significa tener una clara visión sobre la Educación General. Consiste en explicar cómo y por qué las universidades escogen sus prioridades formativas. Idealmente el estudiantado debería comprender la forma en que cada sesión de clase contribuye con los propósitos de aprendizaje y cómo los objetivos de los cursos se relacionan con los propósitos del plan de estudio. Es evidente que un pro- grama de Educación General que posea esta cualidad debe mantener una adecuada comunicación con el estudiantado. Esta claridad de visión puede venir acompañada de programas de orientación que permita al estudiantado reflexionar sistemáticamente sobre sus opciones educativas y las intenciones que las sustentan.

Con sobradas razones, el autor nos advierte que para lograr el compromiso con la coherencia educativa debemos cuidarnos de no buscarla endosando a los estudiantes la responsabilidad para lograrla. Sostiene que solo la estructura del programa de Educación General y el proceso de enseñanza por parte del profesorado involucrado en él permiten al estudiantado comprender el racional detrás de los requisitos que se deben cumplir para alcanzar sus objetivos. Si esto no ocurre, los estudiantes pueden percibir a la Educación General como un conjunto de cursos inconexos que deben tomar fuera de su especialidad y no les prestarán importancia.

A través de las experiencias de Saint Louis Community College y Bard College, el lector puede encontrar suficientes argumentos para convencerse de la necesaria coherencia que debe poseer un programa de Educación General eficiente. En el caso de Bard College, los estudiantes de nuevo ingreso toman, tres semanas antes de iniciar el primer semestre, un taller de lectura, discusión y escritura que les ayuda a entender las metas y los resultados que persigue la formación en esa institución. Además, durante el primer año, participan en seminarios para conocer las nociones intelectuales, artísticas y culturales que son la base de los Estudios Generales; luego, en su segundo año, mediante un programa especial, los estudiantes reflexionan sobre su experiencia educativa a medida que van tomando cursos de su especialidad y, en su último año, tienen la obligación de participar en un proyecto que une los cuatro años de experiencia de aprendizaje en la universidad.

El tercer principio que caracteriza a los buenos programas de Educación General lo constituye su dedicación para formar ciudadanos responsables. En un primer momento, este propósito se pretendía alcanzar asumiendo que los programas basados en valores eran suficientes para que el estudiantado actuara con responsabilidad social al egresar de las universidades. Sin embargo, en la actualidad, se ha incorporado una perspectiva más abarcadora que permite a los estudiantes desarrollar valores basados en informaciones y autoreflexiones a partir de experiencias relacionadas con el trabajo comunitario dentro y fuera del campus universitario.

En este sentido, se reivindica el compromiso de muchos programas con los proyectos de trabajo comunitario como cursos en sí mismos o unidos a clases ya existentes. Cobra relevancia el llamado aprendizaje en el servicio. Se trata de una forma de educación experimental que se caracteriza por la participación del estudiante en una actividad organizada de servicio que se conecta a resultados de aprendizajes específicos, cumple con las necesidades identificadas de la comunidad y provee tiempo estructurado para la reflexión de los estudiantes y la conexión de la experiencia de aprendizaje en el servicio. El libro provee evidencias exitosas de Michigan State University y de Rhodes College en Tennesse, Estados Unidos. 
Los autores del libro reseñado ponen de relieve que formar ciudadanos socialmente responsables no es tarea exclusiva del profesorado de los programas de Educación General sino que corresponde también a los docentes de las especialidades ya que muchos de estos cursos de trabajo comunitario o de aprendizaje en el servicio pueden llevarse a cabo en los últimos años de los estudios universitarios.

Las instituciones de educación superior que ofrecen programas de Estudios Generales ejemplares aprecian el valor educativo de lo cocurricular, conectándolo con lo curricular, como es le caso de Willamette University. Este tipo de institución ha demostrado prestar la suficiente atención a la experiencia estudiantil fuera de las aulas, la cual comprende una amplia gama de actividades como deportes, arte y cultura, asociaciones estudiantiles, debates, conferencias y seminarios, periódicos, Modelo de las Naciones Unidas y otras que forman parte de la vida del campus universitario. En numerosos campus de instituciones estadounidenses, como por ejemplo en la Universidad de Texas, El Paso, ya existe un documento que transcribe como parte del record estudiantil todas las actividades co-curriculares en las que participan sus alumnos.

El autor finaliza este capítulo recordando que un sólido programa de Estudios Generales comienza y termina con el profesorado; sin embargo, se requiere de un liderazgo visionario que abarque a todos los integrantes de las instituciones: estudiantado, profesorado y administradores. Gaston afirma que para que un programa sea efectivo debe encarnar dos principios relacionados entre sí: primero, cualquier reforma curricular que pretenda ser exitosa debe gozar de un respaldo visible del liderazgo administrativo de la universidad y, segundo, el liderazgo estudiantil y profesoral debe tomar esas reformas seriamente planificando enfoques que conciten la aprobación de sus colegas, anticipando, tendiendo puentes o eludiendo los baches que se puedan producir y dedicándose en persona al seguimiento necesario.

El capítulo 3 titulado Intencionalidad inicia la tercera parte del libro. En él la idea principal que lo permea es que los programas de Educación General deben reflejar intencionalidad en todos los niveles. La intencionalidad se asocia a aquellos programas que están deliberadamente organizados para ayudar al estudiantado en su tránsito del bachillerato a la universidad y que los guían a través del trabajo, cada vez más exigente, de las asignaturas que está conectado con la dirección de sus estudios principales, con las experiencias co-curriculares y con sus aspiraciones personales. Esta característica debe estar centrada en el estudiantado, sus necesidades y sus resultados. Los programas exitosos toman conciencia de cómo aprenden los estudiantes y de su diversidad.

En este acápite se resalta la importancia del primer año y su conexión con las asignaturas de la carrera que se estudia. Esta primera etapa debe contribuir a que el estudiantado defina sus metas y asuma responsabilidad en lograrlas. Es importante resaltar que los programas diseñados intencionalmente se preocupan por lo que ocurre no solo en el primer año, sino también en cada uno de años de los estudios universitarios. En este sentido, cada docente necesita trabajar para que sus estudiantes puedan integrar el trabajo que hacen y construir coherentemente sus aprendizajes de curso en curso y de un año a otro. En efecto, se requiere coherencia a lo interno de cada curso y entre los cursos para lograr verdadera intencionalidad y dirección.

Este capítulo nos recuerda que el profesorado que lucha por la intencionalidad haría bien en revisar todos los paradigmas dominantes en el currículo tales como: los supuestos en los prerrequisitos, el significado de los nombres y la numeración de los cursos y la forma en que la asignación de los créditos de cada curso está alineada con los trabajos demandados en las asignaturas y con los resultados obtenidos en ellas.

Alineación con las especialidades es el nombre que lleva el cuarto capítulo. Está escrito por Karen Maitland Schiling y Dwight Smith. Estos autores argumentan a favor de un compromiso institucional que promueva la coherencia curricular entre los estudios generales y las especialidades. Es decir, la Educación General resulta más eficaz si se encuentra bien alineada con las especialidades.

En una encuesta realizada en 2006 por la Asociación de Colegios y Universidades Americanas (AACU), los empleadores han identificado como fundamentales para sus empleados logros de aprendizajes estudiantiles como la competencia comunicativa y el trabajo en equipo. De hecho, estas competencias, propias de los estudios generales, son ahora valoradas con igual relevancia que las adquiridas dentro de las especialidades y que poseen unas características más orientadas a lo profesional laboral.

Los autores ratifican que la alineación de la Educación General y las especialidades es un medio importante para lograr el verdadero fin: el aprendizaje liberal para todos los estudiantes a través de un currículo intencional y bien coordinado. Esta meta requiere un compromiso institucional que Schiling y Smth consideran que se puede conseguir mediante la compresión de la situación del estudiantado, con una apuesta al sistema de educación superior y no para las instituciones aisladas, a través de la incorporación del profesorado de tiempo completo y de carrera docente en las asignaturas de la Educación General y mediante el uso de la evaluación con el fin de entender los logros estudiantiles, para el beneficio de toda la comunidad académica y administrativa.

Los autores nos alertan sobre los nuevos retos que aparecen en las Instituciones de Educación Superior (IES) y que hay que tener presentes en estos momentos de sinergia entre los estudios generales y las especialidades. La movilidad estudiantil se ha incrementado, haciendo que un estudiante no complete todos los cursos de sus estudios generales en una sola institución. Hay estudiantes que se trasladan de colegios comunitarios a otras IES antes de llegar a la institución donde finalmente obtendrán su titulación. Estas transferencias hacen necesario crean alianzas entre las instituciones para la homologación de los cursos incluidos en planes de estudio diversos. No obstante, advierten los autores, esta posibilidad no debe impedir que la alineación que se está llevando a cabo entre los estudios generales y las especialidades se deje a un lado o pierda importancia en el debate académico de las universidades. El reto implica al sis- 
tema de educación superior en su conjunto ya que las instituciones no pueden, dado el contexto, trabajar aisladas.

Alcanzar la coherencia y la alineación entre la Educación General y las especialidades o carreas requiere que se tomen en cuenta, además, la preparación del estudiantado y sus expectativas. No siempre los estudiantes perciben la relación que pudiera existir entre estos dos tipos de enfoques educativos; por este motivo, Schiling y Smith consideran importante que estas expectativas se hagan explícitas a través del currículo. Un ejemplo claro de este caso, lo constituye los resultados de aprendizaje en redacción que se quieren lograr en los primeros años en la universidad y los que se pretenden conseguir en las especialidades. Es necesario que ambas etapas compartan una misma visión sobre la escritura y que la misma sea reforzada tanto en el primer año como en las disciplinas. Existen diversos recursos para lograr dicha coherencia; los mapas curriculares y las rúbricas evaluativas son buenos ejemplos.

La evaluación es también otro medio que los autores presentan como un factor que puede contribuir a la alineación entre la Educación General y las especialidades. La información obtenida de las diversas fuentes de evaluación debe ser tomada en cuenta para lograr esa coherencia. En este sentido, juegan un papel fundamental los datos que se pueden ofrecer las acreditadoras, sobre todo, cuando son los mismos requerimientos tanto para la Educación General como para las especialidades.

El capítulo 5 es de la autoría de Elizabeth Clark, quien presenta el tema de la pedagogía efectiva en el marco de una sólida Educación General. El capítulo se inicia afirmando que los programas de estudios generales deben incorporar pedagogías efectivas a través de su currículo enfatizando aquellas que prioricen el conocimiento interdisciplinar, coloquen al estudiante como centro del aprendizaje y le permitan reflexionar sobre sus logros de manera significativa.

Además, Clark aborda el tema de las metodologías efectivas a partir de las tecnologías de la información y la comunicación. En este sentido, argumenta que los programas de Educación General exitosos dan seguimiento a los cambios en la tecnología, sopesando las consecuencias de dichos cambios, adoptando las herramientas susceptibles de ser útiles en el presente y siendo receptivos a aquellos medios con capacidad de éxito en el futuro y que, sobre todo, mantiene al estudiantado como centro del aprendizaje.

El capítulo concluye afirmando que el profesorado que ha sido reconocido como exitoso en el uso de metodologías efectivas es aquel que reflexiona e investiga sobre su propia práctica. Los graduados de programas sólidos en estudios generales concuerdan que el modelo de una comunidad de aprendizaje promueve la reflexión sobre la práctica docente, anima la colaboración interdisciplinaria y apoya la evaluación de la efectividad didáctica.
El capítulo 6 de este libro lleva por título "Evaluación". Su autora, Peggy Maki, afirma que los programas de Educación General deben documentar su eficacia y demostrar un compromiso dinámico para la mejora continua a través de la articulación clara de los resultados del aprendizaje y la evaluación en relación con los dichos resultados. Como toda práctica de los programas exitosos, la evaluación debe centrase en el estudiantado para que su aprendizaje sea cada vez más enriquecedor.

Maki entiende que los cuatro dominios propios de la Educación General, a saber: conocimiento de las culturas humanas y del mundo físico y natural, habilidades intelectuales y prácticas, responsabilidad personal y social y aprendizaje integral y aplicado deben guiar la evaluación de este tipo de educación. La autora enfatiza que hay que orientar al estudiantado sobre los resultados de estas dimensiones durante los cuatro años de sus estudios de pregrado y que se deben propiciar múltiples oportunidades para aprender, reaprender y practicar esos resultados si se desea que se conviertan en aprendizajes duraderos.

Una estrategia para evaluar la coherencia y la intencionalidad de los estudios generales a lo largo de toda educación universitaria lo constituye la elaboración de mapas curriculares y co-curriculares. Se trata de un recurso que nos habla de la intencionalidad de la formación del estudiantado, los énfasis dados a las diversas áreas del saber y a los estudios generales, la trayectoria prevista para lograr los resultados de aprendizajes esperados, el peso que se le asigna a la práctica versus la teoría y todos los demás elementos que radiografían el currículo universitario.

La autora explica que los programas de Educación General que son exitosos proveen oportunidades para que el estudiantado reflexione sobre su aprendizaje evaluando sus fortalezas y debilidades. En este sentido, son de especial ayuda los portafolios que permiten describir cómo trabajos específicos representan logros y progresos en torno a los resultados institucionales de los estudios generales. Este tipo de evaluación También favorece el involucramiento del estudiante en su propio aprendizaje. Maki afirma que los modelos de evaluación más poderosos para la Educación General son flexibles y personalizados, lo que permite al estudiantado a demostrar su progreso cuando están listos para ello en lugar del tiempo predeterminado de un examen general. En este sentido, favorece el uso de rúbricas evaluativas y sugiere las elaboradas por la AAC\&U.

A través de todo el capítulo, el lector llega a la conclusión de que se requiere evaluación directa e indirecta para determinar la eficacia de la Educación General y que su objetivo primordial es el uso de los datos obtenidos para mejorar el aprendizaje estudiantil. Todo esto conlleva la presencia de un profesorado que se convierte también a aprendiz de su accionar educativo ya que todo sólido programa de Educación General construye ciclos de indagación acerca de la eficacia de sus prácticas. 
Cabe mencionar que, a partir del capítulo 2, donde proliferan los ejemplos de sólidos programas de Educación General, los autores de los demás acápites organizan sus ideas entre características que consideran que los programas de estudios generales debían tener y realidades concretas que ejemplifican cualidades de programas exitosos. Es decir, el lector puede percibir que los principios de prácticas efectivas se mueven entre el deber ser y la realidad concreta. Hubiese sido mejor que las ideas expresadas en cada capítulo se presentaran siempre con instituciones que lleven a cabo dichos principios.

La cuarta parte del libro reseñado que lleva por nombre "La sostenibilidad de los programas de Educación General" concluye con el capítulo 7. No en vano el capítulo se titula "Compromiso institucional". Su autor, Paul Gaston, quiere que el lector infiera que no existe un sólido programa de estudios generales que sea sostenible si la institución universitaria no asume, a todos los niveles, la responsabilidad de apoyar este tipo de educación. En este sentido, afirma que "Los programas de Educación General que crecen continuamente en fortaleza y efectividad dependen del amistoso escrutinio y el dedicado apoyo del profesorado, los administradores y la comunidad universitaria como un todo" (p.51).

En este último capítulo Gaston dedica una buena parte de su reflexión sobre el compromiso institucional al profesorado universitario, lo que resulta uno de los aspectos más positivos de este libro. Afirma que cada miembro del cuerpo docente, cualquiera que sea su campo de experiencia, contribuye al aprendizaje liberal de cada estudiante, para bien o para mal. Este reconocimiento, sigue diciendo el autor, se hace más real con ejemplos en que el profesorado no solo enseña las características de este tipo de aprendizaje, sino que lo valora de manera especial y lo vivencia en la cotidianidad.

Es interesante que Gaston no deja de mencionar el caso opuesto: el de aquellos docentes que manifiestan desprecio por los objetivos del aprendizaje liberal; sin embargo, los considera menos peligrosos para el estudiantado que miembros del cuerpo docente que a pesar de su autoridad y camuflajeado atractivo personal presentan un enfoque estrecho y antiliberal del aprendizaje, sobre todo aquellos docentes que se concentran en los contenidos de las asignaturas y dejan de explicar las consecuencias éticas relacionadas con su disciplina. En este sentido, el autor termina insistiendo en la responsabilidad que tiene la institución de que los cursos relacionados con los estudios generales sean impartidos por un profesorado cuyo quehacer intelectual y cuyo accionar personal sirvan para modelar los principios del aprendizaje liberal.
Asimismo, este último capítulo contiene ocho perspectivas que el autor considera valiosas para las instituciones que se comprometen con el aprendizaje liberal. Ellas son:

1. Romper con las barreras disciplinares en apoyo de la transdisciplinariedad.

2. Ejemplificar el compromiso con la Educación General entre sus colegas.

3. Establecer con claridad los objetivos de la educación liberal en cada curso.

4. Ser flexible y relevante.

5. Permitir que los estudiantes se apropien de su aprendizaje.

6. Promover el aprendizaje liberal en la vida diaria.

7. Destacar las relaciones en temas y disciplinas.

8. Servir de ejemplo al estudiantado sobre el aprendizaje a lo largo de la vida.

Estas perspectivas pueden llevar a las instituciones de educación general a buscar un perfil organizacional que las distingue y las singularice en su accionar educativo, con el fin de lograr el propósito primordial del aprendizaje liberal que los egresados pueden ejercer sus carreras con éxito y profesionalidad al tiempo que viven de forma responsable social y personalmente.

Es imposible dejar de reiterar que el contexto en que se enmarcan los ejemplos citados en este libro responde a una realidad que difiere de la dominicana. No obstante, lo que en un principio puede verse como una limitación, también podría visualizarse como una perspectiva distinta para reflexionar sobre formas innovadoras de abordar la Educación General; asimismo, este documento puede muy bien servir como marco referencial para reformas educativas dentro de los estudios generales ya que expone con una actualizada fundamentación conceptual y con una adecuada claridad expositiva los principios que subyacen a los programas de Educación General que han dado frutos de un verdadero aprendizaje liberal. 